\title{
Lithiation Directed by Amino Alkoxydes. Application to the Synthesis of New Disubstituted
}

Dihydrodipyridopyrazines

Irina-Claudia Grig-Alexa ${ }^{1,2}$, Adriana-Luminița Fînaru ${ }^{2}$, Paul Caubère ${ }^{1}$ and Gérald Guillaumet ${ }^{1 *}$

\section{Demande de}

Résultats

Dernière modification : Tuesday, 20 June 2006, 10H

SUZENET , Equipe

$A I C 335$ (31044)<smiles>Cc1ccnc2c1N(C)c1c(C=O)ccnc1N2C</smiles>

Passage : $16 / 06 / 2006$

Product 2

$\mathrm{T}^{\circ}$ Introduction : $60^{\circ} \mathrm{C}$

Structure non affichée

Opérateur : Marion MILLOT

Composé principal

M+.

Masse Théorique : 254.11676

(C14 H14 N4 O )

Masse Trouvée : $254.1166(0 \mathrm{ppm})$

\section{Retour au tableau global des résultats}

Les résultats sont sous réserve de vérification par Pierre GUENOT, directeur du CRMPO

Ce service d'accès est toujours en amélioration.

Merci de signaler tout dysfonctionnement au WEBMASTER

Pour toute question ou problème concernant ce site Web, envoyez un email à guenot@univ-rennesl.fr

Copyright 2002 CRMPO. Tous droits réservés 
Demande de

Résultats

\begin{tabular}{l|c|l|l}
$\begin{array}{l}\text { Listing } \\
\text { complet }\end{array}$ & $\underset{\text { Mini Rapport }}{\text { résumé par groupe de 10 }}$ & Déconnexion & Votre Compte \\
\hline
\end{tabular}

Dernière modification : Friday, 16 June 2006, $10 \mathrm{H}$

SUZENET , Equipe

AIC 382 (31045)<smiles>CN1c2nccc(C=O)c2N(C)c2nccc([SiH3])c21</smiles>

Passage : 01/05/2006

Structure non affichée

$\mathrm{T}^{\circ}$ Introduction : $90^{\circ} \mathrm{C}$

Product 3

Opérateur : Fabian LAMBERT

Composé principal

$\mathrm{M}+$.

(C16 H20 N4 O Si )

Masse Théorique : 312.14064

Masse Trouvée : $\mathbf{3 1 2 . 1 3 8 5}(6 \mathrm{ppm})$

[M-.CH3 ]+

Masse Théorique : 297.11716

(C15 H17 N4 O Si )

Masse Trouvée : $297.1164(2 \mathrm{ppm})$

\section{Retour au tableau global des résultats}

Les résultats sont sous réserve de vérification par Pierre GUENOT, directeur du CRMPO

Ce service d'accès est toujours en amélioration.

Merci de signaler tout dysfonctionnement au WEBMASTER

Pour toute question ou probleme concemant ce site Web, envoyez un email à guenot@univ-rennes1.fr.

Copyright 92002 CRMPO. Tous droits réservés 
Demande de

Résultats

\begin{tabular}{|c|c|c|c|}
\hline $\begin{array}{l}\text { Listing } \\
\text { complet }\end{array}$ & $\begin{array}{c}\text { Mini Rapport } \\
\text { résumé pargroupe de 10 }\end{array}$ & Déconnexion & Votre Compte \\
\hline
\end{tabular}

Dernière modification : Tuesday, 20 June 2006, 10H

SUZENET , Equipe

AIC 411 (31052)<smiles>CN1c2nccc(C=O)c2N(C)c2nccc(I)c21</smiles>

Product 5

Passage : $16 / 06 / 2006$

Structure non affichée

$\mathrm{T}^{\circ}$ Introduction : $115^{\circ} \mathrm{C}$

Opérateur : Marion MILLOT

Composé principal

M+.

(C13 H11 N4 O I )

Masse Théorique : $\mathbf{3 6 5 . 9 9 7 7 6}$

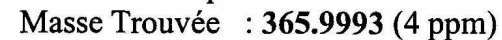

\section{Retour au tableau global des résultats}

Les résultats sont sous réserve de vérification par Pierre GUENOT, directeur du CRMPO

Ce service d'accès est toujours en amélioration.

Merci de signaler tout dysfonctionnement au WEBMASTER

Pour toute question ou problème concernant ce site Web, envoyez un email à guenot@univ-rennes1.fr.

Copyright 12002 CRMPO. Tous droits réservés 
Demande de

Résultats

\begin{tabular}{|l|c|c|c|}
\hline $\begin{array}{l}\text { Listing } \\
\text { complet }\end{array}$ & $\begin{array}{c}\text { Mini Rapport } \\
\text { résumé par groupe de 10 }\end{array}$ & Déconnexion & Votre Compte \\
\hline
\end{tabular}

Dernière modification : Monday, 5 June 2006, 10H

SUZENET , Equipe

AIC 399 (31046)

Passage : 03/06/2006

Structure non affichée

$\mathrm{T}^{\circ}$ Introduction : $\mathbf{1 3 5}^{\circ} \mathrm{C}$<smiles>Cc1ccnc2c1N(C)c1c(C=O)ccnc1N2C</smiles>

Product 6

Opérateur : Marion MILLOT

Composé principal

M+.

Masse Théorique : $\mathbf{2 6 8 . 0 9 6 0 3}$

(C14 H12 N4 O2)

Masse Trouvée : $268.0951(3 \mathrm{ppm})$

\section{Retour au tableau global des résultats}

Les résultats sont sous réserve de vérification par Pierre GUENOT, directeur du CRMPO

Ce service d'accès est toujours en amélioration.

Merci de signaler tout dysfonctionnement au WEBMASTER

Pour toute question ou problème concernant ce site Web, envoyez un email à guenot@univ-rennesl.fr.

Copyright $\odot 2002$ CRMPO. Tous droits réservés 
Demande de

Résultats

\begin{tabular}{l|c|c|c|}
$\begin{array}{l}\text { Listing } \\
\text { complet }\end{array}$ & $\underset{\text { Mini Rapport }}{\text { résumé par groupe de 10 }}$ & Déconnexion & Votre Compte \\
\hline
\end{tabular}

Dernière modification : Friday, 16 June 2006, 10H

SUZENET , Equipe

$A I C 405$ (31050)

Passage : $15 / 06 / 2006$

Structure non affichée

$\mathrm{T}^{\circ}$ Introduction : $110^{\circ} \mathrm{C}$<smiles>CN1c2nccc(S(C)(=O)=O)c2N(C)c2nccc(C=O)c21</smiles>

Product 7

Opérateur : Fabian LAMBERT

Composé principal

$\mathrm{M}+$.

Masse Théorique : $\mathbf{2 8 6 . 0 8 8 8 3}$

(C14 H14 N4 O S )

Masse Trouvée : $286.0879(3 \mathrm{ppm})$

[M-.CH3 ]+

(C13 H11 N4 O S )

Masse Théorique : $\mathbf{2 7 1 . 0 6 5 3 6}$

Masse Trouvée : $271.0668(5 \mathrm{ppm})$

\section{Retour au tableau global des résultats}

Les résultats sont sous réserve de vérification par Pierre GUENOT, directeur du CRMPO

Ce service d'accès est toujours en amélioration.

Merci de signaler tout dysfonctionnement au WEBMASTER

Pour toute question ou problème concernant ce site Web, envoyez un email à guenot@univ-rennes1.fr

Copyright $\$ 2002$ CRMPO. Tous droits reservés 
Demande de

Résultats

\begin{tabular}{l|c|c|c|}
$\begin{array}{l}\text { Listing } \\
\text { complet }\end{array}$ & $\begin{array}{c}\text { Mini Rapport } \\
\text { résumé par groupe de 10 }\end{array}$ & Déconnexion & Votre Compte \\
\hline
\end{tabular}

Dernière modification : Tuesday, 20 June 2006, $10 \mathrm{H}$

SUZENET , Equipe

AIC 407 (31051)

$\begin{array}{ll}\text { Structure non affichée } & \text { Passage }: 16 / 06 / 2006 \\ & \mathrm{~T}^{\circ} \text { Introduction : } 180^{\circ} \mathrm{C} \\ & \text { Opérateur : Marion MILLOT }\end{array}$

Masse Théorique : $\mathbf{4 2 2 . 1 7 4 2 8}$

Composé principal

$\mathrm{M}+$.

(C26 H22 N4 O2)

Masse Trouvée : $422.1747(1 \mathrm{ppm})$

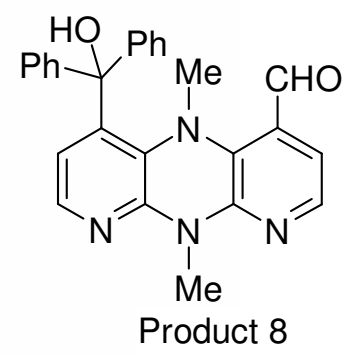

\section{Retour au tableau global des résultats}

Les résultats sont sous réserve de vérification par Pierre GUENOT, directeur du CRMPO

Ce service d'accès est toujours en amélioration.

Merci de signaler tout dysfonctionnement au WEBMASTER

Pour toute question ou problème concernant ce site Web, envoyez un email a guenot@univ-rennes1.fr

Copyright 02002 CRMPO. Tous droits réservés 


\begin{tabular}{l|c|c|c|}
$\begin{array}{l}\text { Listing } \\
\text { complet }\end{array}$ & $\begin{array}{c}\text { Mini Rapport } \\
\text { résumé par gruupe de 10 }\end{array}$ & Déconnexion & Votre Compte \\
\hline
\end{tabular}

Dernière modification : Friday, 16 June 2006, $10 \mathrm{H}$

SUZENET , Equipe

AIC 443 (31056)<smiles>CCCCN1c2nccc(C=O)c2N(C(=O)c2ccccc2)c2nccc(C=O)c21</smiles>

Product 9

Structure non affichée

Passage : 14/06/2006

$\mathrm{T}^{\circ}$ Introduction : $90^{\circ} \mathrm{C}$

Opérateur : Fabian LAMBERT

Composé principal

M+.

(nt=H )

Masse Théorique : $\mathbf{e}=\mathbf{3 5 2 . 1 8 9 8 8}$

Masse Trouvée : FK. $(o=352.18993$ ppm)

[M-.tBu ]+

Masse Théorique : $\mathbf{2 9 5 . 1 1 9 5 0}$

(C16 H15 N4 O2)

Masse Trouvée : $295.1195(0 \mathrm{ppm})$

\section{Retour au tableau global des résultats}

Les résultats sont sous réserve de vérification par Pierre GUENOT, directeur du CRMPO

Ce service d'accès est toujours en amélioration.

Merci de signaler tout dysfonctionnement au WEBMASTER

Pour toute question ou problème concernant ce site Web, envoyez un email à guenot@univ-rennes1.fr

Copyright 2002 CRMPO. Tous droits réservés 


\begin{tabular}{l|l|l|l}
$\begin{array}{l}\text { Listing } \\
\text { complet }\end{array}$ & $\underset{\text { Mini Rapport }}{\text { résumé par groupe de 10 }}$ & Déconnexion & Votre Compte \\
\hline
\end{tabular}

Dernière modification : Tuesday, 20 June 2006, 10H

SUZENET , Equipe

$A I C 442$ (31055)

Passage : 16/06/2006

Structure non affichée

$\mathrm{T}^{\circ}$ Introduction : $\mathbf{7 0}^{\circ} \mathrm{C}$

Opérateur : Marion MILLOT<smiles>C[SiH3]c1ccnc2c1N(CC(C)C)c1c(C=O)ccnc1N2C(=O)c1ccccc1</smiles>

Product 10
Composé principal

M+.

(C22 H32 N4 O Si )

[M-.C4H9 ]+

(C18 H23 N4 O Si )
Masse Théorique : $\mathbf{3 9 6 . 2 3 4 5 4}$

Masse Trouvée : $396.2351(1 \mathrm{ppm})$

Masse Théorique : $\mathbf{3 3 9 . 1 6 4 1 1}$

Masse Trouvée : $339.1636(1 \mathrm{ppm})$

\section{Retour au tableau global des résultats}

Les résultats sont sous réserve de vérification par Pierre GUENOT, directeur du CRMPO

Ce service d'accès est toujours en amélioration.

Merci de signaler tout dysfonctionnement au WEBMASTER

Pour toute question ou problème concernant ce site Web, envoyez un email à guenot@univ-rennesl.fr.

Copyright 02002 CRMPO. Tous droits réservès 